\title{
RESPUESTA DEL PLÁTANO A LA FERTILIZACIÓN CON P, K Y S DURANTE EL PRIMER CICLO PRODUCTIVO ${ }^{1}$
}

\author{
Parménides Furcal-Beriguete ${ }^{2}$, Alejandro Barquero-Badilla ${ }^{3}$
}

\section{RESUMEN}

Respuesta del plátano a la fertilización con $\mathbf{P}, \mathbf{K}$ y $\mathbf{S}$ durante el primer ciclo productivo. El objetivo de este trabajo fue evaluar la respuesta en crecimiento y rendimiento de la primera generación del plátano (Musa AAB) a la aplicación de fósforo, potasio y azufre . El estudio se estableció en La Fortuna, San Carlos, Costa Rica durante el periodo 2008 a 2010. El suelo donde se establecieron los experimentos es del orden inceptisol, formado por materiales volcánicos, de fertilidad media y textura franco arcillosa. Se aplicaron 0,125 , $250,375 \mathrm{~kg} / \mathrm{ha} \mathrm{de} \mathrm{K}_{2} \mathrm{O}, 0$ y $70 \mathrm{~kg} / \mathrm{ha}$ de $\mathrm{P}_{2} \mathrm{O}_{5}$ en el ensayo 1 , mientras que en el ensayo 2 se aplicaron los mismos niveles de $\mathrm{K}_{2} \mathrm{O}$, además de 0 y $30 \mathrm{~kg} /$ ha de azufre (S). La siembra se realizó en abril y mayo en 2008 y 2009, respectivamente, con cormos del cultivar Curraré semi gigante con 2380 y 2223 plantas por hectárea, en cada uno de los años. No hubo diferencia estadística a las dosis de $\mathrm{K}_{2} \mathrm{O}$ en la primera cosecha (2009), pero sí en la segunda (2010) en las variables circunferencia y altura de pseudotallo a los cuatro y siete meses en el experimento 2, en peso del racimo, longitud y calibre del dedo central de la segunda mano. Para estas tres variables, con $375 \mathrm{~kg}$ de $\mathrm{K}_{2} \mathrm{O}$, los valores fueron: $13,17 \mathrm{~kg}, 28,81 \mathrm{~cm}$ y 62,62 treintaidosavos de pulgada, respectivamente. Solo hubo respuesta a la utilización de $\mathrm{P}_{2} \mathrm{O}_{5}$ en altura y circunferencia a los cuatro meses $(1,32 \mathrm{~m}$ y $0,39 \mathrm{~m})$, no así para el azufre. La absorción total de K, P y S al momento de la cosecha fue de 237,$73 ; 10,83$ y $7,44 \mathrm{~kg} / \mathrm{ha}$, respectivamente.

Palabras claves: Musa AAB, altura de pseudotallo, absorción de $\mathrm{K}, \mathrm{P}$ y S en Musa AAB, cultivar de plátano Curraré.

\begin{abstract}
Response of plantain to fertilization with $P, K$ and $S$ during the first productive cycle. The objective of this work was to evaluate the growth and yield response of the first reproductive cycle of plantain (Musa AAB) to the application of phosphorus, potassium and sulfur. The study was established in La Fortuna, San Carlos, Costa Rica. The soil where the experiments were established is an Inceptisol, formed by volcanic materials, with medium fertility and clayloam texture. Doses of $0,125,250,375 \mathrm{~kg} / \mathrm{ha}$ of $\mathrm{K}_{2} \mathrm{O}, 0$ and $70 \mathrm{~kg} / \mathrm{ha}$ of $\mathrm{P}_{2} \mathrm{O}_{5}$ were applied in trial 1, while in trial 2 were applied the same levels of $\mathrm{K}_{2} \mathrm{O}$ were used, in addition to 0 and $30 \mathrm{~kg} / \mathrm{ha}$ of sulfur (S). The sowing was made in April and May of 2008 and 2009, respectively, with corms of the cultivar Curraré "Semi Giant", with a density of 2.380 and 2.223 plants per hectare, in each year. There was no statistical response to $\mathrm{K}_{2} \mathrm{O}$ doses in the first harvest, but differences were obtained in the second trial (2010) for the variables pseudostem girth and height at four and seven months in experiment 2 , and bunch weight, length and thickness of the middle finger of the second hand. For these three variables, with $375 \mathrm{~kg}$ of $\mathrm{K}_{2} \mathrm{O}$, the values were: $13.17 \mathrm{~kg}, 28.81 \mathrm{~cm}$ and 62.62 inch treintaidosavo respectively. There was only response to the use of $\mathrm{P}_{2} \mathrm{O}_{5}$ in height and girth at four months $(1.32 \mathrm{~m} 0.39 \mathrm{~m})$, not so for $\mathrm{S}$. The total absorption of $\mathrm{K}, \mathrm{P}$ and $\mathrm{S}$ at the time of harvest was $237.73,10.83$ and $7.44 \mathrm{~kg}$ / ha, respectively.
\end{abstract}

Keywords: Musa AAB nutrition, pseudostem height, absorption of K, P and S, cultivar Curraré.

\footnotetext{
Recibido: 31 de enero, 2013. Aceptado: 28 de octubre, 2013. Parte del proyecto número 5402-2151-7801, financiado por la Vicerrectoría de Investigación y Extensión del Instituto Tecnológico de Costa Rica (ITCR).

2 Instituto Tecnológico de Costa Rica (ITCR), Escuela de Agronomía, Sede San Carlos, Alajuela, Costa Rica. Apartado: 223-21001. C. Quesada.pafurcal@itcr.ac.cr, pfurcal@hotmail.com (autor para correspondencia).

3 Bayer Crop Science S.A. Eurocenter II, Barreal de Heredia, Costa Rica. alejandro.barquero@bayer.com, alejandro.csh@hotmail.com
}

(†) $\odot$ (C) 2013 Agronomía Mesoamericana es desarrollada en la Universidad de Costa Rica y se encuentra licenciada con Creative EY No ND Commons Reconocimiento-NoComercial-SinObraDerivada 3.0 Costa Rica. Para más información escríbanos a pccmca@ucr.ac.cr 


\section{INTRODUCCIÓN}

A raíz de la presencia de factores agroecológicos favorables y nichos de mercado, el plátano se ha convertido en una alternativa de producción en la zona Huetar Norte y Atlántica de Costa Rica, que son las zonas de mayor auge del país, la primera con el $25 \%$ de la producción nacional y la segunda entre el 50 y 51\% (Barrientos y Chaves 2008, Navarro et al. 2009).

El grado de respuesta del plátano a la fertilización, de igual manera que otros muchos cultivos, no es uniforme en todos los suelos, sino que depende del contenido inicial o potencial nutricional de estos (Combatt et al. 2004, Palencia et al. 2006). Bajo esa consideración, se debe recomendar, aplicación de nutrientes en forma más eficiente y económica, utilizando los resultados de análisis de suelos y los niveles óptimos de los elementos prioritarios para los rendimientos de un cultivo determinado. Por consiguiente, es importante conocer la fertilidad de los suelos y definir los niveles más adecuados de los elementos importantes para el rendimiento económico de un cultivo de interés. Actualmente, se conocen las características generales de los suelos de la zona norte, encontrándose que el fósforo es uno de los elementos más limitantes; sin embargo, no se conocen los niveles de potasio, como uno de los elementos más importantes en el rendimiento económico del cultivo de plátano en el cantón de San Carlos y cantones vecinos.

El fósforo $(\mathrm{P})$ es deficiente en la zona del estudio, a la vez el cultivo de plátano presenta baja respuesta a este elemento (Mena 1997, Espinosa y Belalcázar 1998, López 2002). También, es un elemento que, pese a no haber sido estudiado en la zona, ha sido usado ampliamente en las plantaciones de plátano y otros cultivos conjuntamente con el nitrógeno y el potasio; pero a diferencia del fósforo, el potasio es el elemento de mayor absorción por este cultivo, y conjuntamente con el nitrógeno es limitante para el rendimiento y la calidad (Espinosa y Belalcázar 1998, Combatt et al. 2004). En ese sentido, la fertilización con fósforo y potasio se consideró como alternativa de investigación en el manejo general del cultivo de plátano, con el fin de mejorar la productividad, calidad y economía de este cultivo.

Existe respuesta a la aplicación de $30 \mathrm{~kg} / \mathrm{ha}$ de azufre (S), aunque depende de cada suelo (Palencia et al. 2006). Espinosa y Belalcázar (1998) también encontraron respuesta del plátano a este elemento. El azufre es un nutrimento ausente en los programas de fertilización utilizados por los agricultores dedicados al cultivo de plátano; sin embargo, en suelos cuyos materiales son de origen volcánico se ha observado, que cuando se suministra $\mathrm{P}$ y B, se aumenta la probabilidad de manifestarse las deficiencias de $\mathrm{S}$ (Bornemisza 1990); por otra parte, se presentan en la zona ciertos síntomas visibles que sugieren deficiencias de $S$; por lo que se decidió estudiar si hay respuesta a este elemento. El objetivo del estudio fue evaluar la respuesta en crecimiento y rendimiento del primer ciclo (planta madre) del cultivo de plátano cultivar Curraré "semi gigante" a la fertilización con $\mathrm{P}, \mathrm{K}$ y $\mathrm{S}$.

\section{MATERIALES Y MÉTODOS}

\section{Ubicación del estudio, siembra y manejo del cultivo}

En la Fortuna de San Carlos, Costa Rica se realizaron dos experimentos de fertilización en el cultivo de plátano en dos siembras consecutivas en los años 2008 y 2009, cuyas cosechas se efectuaron un año después, 2009 y 2010 respectivamente. Ambos experimentos se instaron en lotes diferentes, muy cerca uno del otro en la misma finca. El suelo en estos lotes corresponde al orden inceptisol formado por materiales aluviales de origen volcánico, fertilidad media y textura franco arcillosa. Los análisis de suelos, realizados antes de la siembra, arrojaron pH 5,7 y 5,9, acidez 0,47 y 0,18 $\mathrm{cmol}(+) / 1$, sumatoria de bases 12,6 y $9,07 \mathrm{cmol}(+) / 1$, contenido de potasio (K) 0,36 y $0,38 \mathrm{cmol}(+) / 1$, valores de fósforo $(\mathrm{P}) 4$ y $5 \mathrm{ppm}$, y contenido de materia orgánica de 3,4 y 3,2\% según el año de siembra 2008 y 2009, respectivamente. La caracterización del pH se hizo en agua, la extracción de P y K mediante el método Olsen Modificado, mientras que el procedimiento para la obtención de la acidez, el calcio y el magnesio fue por medio de $\mathrm{KCl} 1 \mathrm{M}$. El área experimental se ubica a $165 \mathrm{msnm}$, en las coordenadas geográficas $10^{\circ} 49^{\prime}$ $\mathrm{N}$ y $84^{\circ} 60^{\prime} \mathrm{O}$, la temperatura en la época del estudio varió entre 22 y $31,5^{\circ} \mathrm{C}$ y la precipitación anual fue de 4463 y $3801 \mathrm{~mm}$ para los períodos de la investigación 2008-2009 y 2009-2010, respectivamente.

La siembra se hizo a doble hilera con cormos seleccionados del cultivar Curraré "semi gigante", el más difundido en la zona. La densidad usada fue de 
2380 y 2224 plantas por hectárea, respectivamente en cada época de siembra. El material de siembra fue extraído de una plantación comercial, el mismo fue seleccionado de tamaño similar y sanidad adecuada, con el propósito de realizar la siembra con cormos relativamente homogéneos y sanos; los cormos fueron tratados por inmersión durante 10 a 15 minutos en una solución con insecticida-nematicida (Oxamyl) a la dosis de $5 \mathrm{ml} / 1$ de agua, contra posible presencia de plagas y prevención de incidencia a temprana edad en el campo; a los tres meses se hizo una aplicación de $2,5 \mathrm{~g} / \mathrm{mata}$ de Terbufos.

Las labores de manejo general del área experimental (control fitosanitario, control de malezas, deshijes, deshojes, entre otras) fueron ejecutadas de acuerdo con la presencia de malezas, ritmo de emisión de hijos, hojas senescentes y enfermas; para el caso de enfermedades, especialmente Sigatoka negra, el control fue preventivo y curativo, el preventivo consistió en deshojas, deshijas y uso de Mancozeb más aceite agrícola a la dosis de $2 \mathrm{~kg} /$ ha y $5 \mathrm{l} / \mathrm{ha}$, respectivamente. Respecto al control curativo se hizo uso de Carbendazina en dos ocasiones $(0,4 \mathrm{l} / \mathrm{ha})$ y una aplicación Metil Tiofanato $(0,5 \mathrm{l} / \mathrm{ha})$.

\section{Tratamientos y diseño experimental}

Los tratamientos usados fueron: factor potasio como $\mathrm{K}_{2} \mathrm{O}$ con cuatro niveles $(0,125,250$ y $375 \mathrm{~kg} / \mathrm{ha})$, factor fósforo como $\mathrm{P}_{2} \mathrm{O}_{5}$ con dos niveles $(0 \mathrm{y} 70 \mathrm{~kg}$ ) ha), mientras que en el factor azufre (S) se usaron los niveles 0 y $30 \mathrm{~kg} / \mathrm{ha}$. El diseño utilizado fue bloques al azar con muestreo en un arreglo factorial $4 * 2$ en los dos experimentos, donde 4 y 2 son los niveles de los factores potasio y fósforo $(\mathrm{K} * \mathrm{P})$ en un experimento, en el otro corresponden a los niveles de potasio y azufre $(\mathrm{K} * \mathrm{~S})$, respectivamente. Cada tratamiento fue repetido cuatro veces para un total de 32 unidades experimentales por ensayo. Como fuente de $\mathrm{K}_{2} \mathrm{O}$ se usó el cloruro de potasio, en tanto que para $\mathrm{P}_{2} \mathrm{O}_{5}$ se usó superfosfato triple y como fuente de $\mathrm{S}$ se aplicó sulfato de amonio. En todas las unidades experimentales se aplicaron 100 $\mathrm{kg}$ de $\mathrm{N} / \mathrm{ha}$; además, en el ensayo $\mathrm{K}_{2} \mathrm{O} * \mathrm{~S}$ se aplicaron $70 \mathrm{~kg}$ de $\mathrm{P}_{2} \mathrm{O}_{5} / \mathrm{ha}$, con la finalidad de prevenir posibles deficiencias de nitrógeno y fósforo y propiciar mejor manifestación de los tratamientos.

El ciclo vegetativo del plátano comprende tres fases fenológicas: vegetativa, reproductiva y productiva
(Belalcázar 1991). De acuerdo con Guerrero (2010) la primera fase comprende desde la emisión de las raíces del cormo hasta los seis meses, cuando inicia la fase reproductiva o floral; esta última dura entre 85 y 90 días, desde la salida del tallo floral a partir del cormo, elevándose a través del pseudotallo y es visible hasta que aparece la inflorescencia, a continuación las brácteas comienzan a abrirse y caerse, exponiendo los dedos. En la fase productiva o fructificación se da la organización de la inflorescencia, se diferencian las flores masculinas y femeninas y se produce el llenado de los frutos que conforman el racimo, finaliza con la cosecha, esta fase dura entre 81 y 90 días (González 1999, Guerrero 2010), comprende parámetros de rendimiento y calidad de la producción.

Desde el punto de vista de programación de la fertilización, interesa la fase vegetativa, que comprende las subfases: brotación, organogénesis y diferenciación floral (Belalcázar 1991). La brotación es el período que inicia cuando aparecen las raíces hasta la emisión de las primeras hojas (alcanza los primeros 30 días), esta es de lenta absorción de nutrimentos. En la subfase organogénesis la mayoría de las raíces salen de la parte superior del cormo, muestra sensibilidad al suministro de elementos minerales y la mayoría de absorción de potasio se da en ella. El desarrollo alcanzado por la planta en esta etapa, influye directamente sobre el número máximo de frutos que van a desarrollarse, aunque también el clima que prevalezca en la fase de floración influye en el rendimiento de la planta (Rodríguez y Guerrero 2002). La subfase diferenciación floral ocurre en un período aproximado entre los seis a siete meses de iniciado el proceso de emisión de hojas. En este momento el meristemo o ápice vegetativo inicia su proceso fisiológico de diferenciación, es decir, se detiene el desarrollo vegetativo para dar paso a una yema floral, la cual dará origen a la inflorescencia y posteriormente, al racimo (Belalcázar 1991).

Desde la brotación hasta la hoja 16 la absorción de nutrimentos es lenta, a partir de este momento la acumulación de estos es alta, período que coincide con el desarrollo de los hijos y previo a la diferenciación floral que se produce alrededor de la aparición de la hoja 22, es decir que la mayor absorción de nutrimentos ocurre entre la hoja 16 y cuando la inflorescencia se hace visible (Sancho 1999).

Los tratamientos fueron aplicados de acuerdo con las fases y la demanda nutricional del cultivo, de 
acuerdo con la propuesta de Palencia et al. (2006) y los resultados de Sancho (1999). De manera tal que todo el $\mathrm{P}$ fue aplicado al mes de la siembra, el $\mathrm{K}_{2} \mathrm{O}$ y el $\mathrm{S}$ se aplicaron de la siguiente manera: $15 \%$ antes de la apertura de la hoja 15 , alrededor de 2,5 meses después de la siembra, $50 \%$ a los cuatro meses y el restante $35 \%$ antes de la emergencia de la inflorescencia que inició a los siete meses.

El análisis de datos se hizo mediante el software estadístico InfoStat (2008). Con este se realizó el análisis de varianza y de las tendencias de los datos, utilizando contrastes polinomiales.

\section{Variables y metodología de toma de datos}

Se midieron las variables altura y circunferencia "grosor" de pseudotallo (AP y GP), el peso del racimo (PR), número de manos por racimo ( $\left.{ }^{\circ} \mathrm{MR}\right)$, número de dedos por racimo $\left(\mathrm{N}^{\circ} \mathrm{DR}\right)$, longitud y calibre del dedo central de la segunda mano (LD) y (CD), respectivamente.

La medición de las variables de respuesta se realizó en las tres plantas centrales de cada unidad experimental en la primera siembra y, en las dos centrales en la segunda. En algunos tratamientos no se alcanzó a cosechar las tres plantas centrales de la primera siembra (2008-2009) debido al volcamiento antes de alcanzar su estado de madurez fisiológica, producto de los vientos y lluvias presentes en diciembre y la presencia de bacteriosis a los cinco meses de edad. Al consultar y analizar esta enfermedad se encontró que corresponde a Dyckeya paradisiaca (Syn. Erwinia chrysanthemi).

Además de las variables anteriores, se tomaron muestras foliares al inicio de la floración en cada una de las unidades experimentales, de las cuales se formaron muestras compuestas en las que se analizó la concentración de nutrimentos en lámina de las hojas $(\mathrm{CNH})$ para cada uno de los tratamientos. Para el análisis de la $\mathrm{CNH}$, se tomó la parte media de la lámina de la tercera hoja numerada en la planta desde arriba hacia abajo.

A las plantas correspondientes a la parcela útil se les midió la altura desde la superficie del suelo hasta el punto de salida de la flor. Además, en la base del pseudotallo se hizo la medida de la circunferencia (5 $\mathrm{cm}$ sobre la superficie del suelo). Estas mediciones se hicieron a los cuatro y siete meses después de la siembra, es decir, después de la tercera fertilización y alrededor de quince días después de haber completado los tratamientos, respectivamente.
En cada racimo de las plantas del área útil de las unidades experimentales y durante cada cosecha, se midieron y anotaron los datos obtenidos de las variables: $\mathrm{PR}, \mathrm{N}^{\circ} \mathrm{MR}, \mathrm{N}^{\circ} \mathrm{DR}, \mathrm{LD}$ y $\mathrm{CD}$. Para obtener estos datos se utilizó balanza, cinta métrica, calibrador en treinta y dosavos de pulgada, y calibrador o "pie de rey". La medición de estas variables se realizó por racimo cosechado en el momento de cada cosecha, en los dos ensayos, tanto en el 2009 como en el 2010. Cuando el período de la cosecha se estabilizó, se tomó una planta en cada unidad experimental con mayor dosis de nutrimentos, es decir plantas que teóricamente estaban "mejor nutridas" (correspondientes al tratamiento 100-70-375-30(S)); estas cuatro plantas se seccionaron en sus diferentes órganos, se pesaron en el campo, se revolvieron y posteriormente se tomaron submuestras de los órganos de las cuales se obtuvo materia seca y análisis químicos, calculando así la absorción total de nutrimentos.

\section{RESULTADOS Y DISCUSIÓN}

Bajo las condiciones de este estudio, en la cosecha 2009, no hubo respuesta a la aplicación de $70 \mathrm{~kg}$ de $\mathrm{P}_{2} \mathrm{O}_{5} /$ ha ni a las dosis de $\mathrm{K}$ estudiadas, excepto para las variables de crecimiento altura y circunferencia del pseudotallo a los cuatro meses de la siembra, las cuales presentaron diferencias al P (AP y GP, $\mathrm{p}=0,0001$ ) y a la interacción P-K (AP, $\mathrm{p}=0,0083 ; \mathrm{GP}, \mathrm{p}=0,0421$ ) (Figuras 1 y 2), pero no a los siete meses, lo cual es indicativo que el fósforo es útil para este cultivo durante la etapa inicial de crecimiento, especialmente para las raíces (Mena 1997), aunque el comportamiento de este elemento en musáceas es muy discutido.

Las variables de altura y circunferencia a los cuatro meses de edad en la cosecha 2009 tuvieron valores promedio entre 1,06 y $1,38 \mathrm{~m}$ de altura y entre 0,33 y $0,40 \mathrm{~m}$ de circunferencia, siendo los más altos cuando se aplicaron las dosis alta de $\mathrm{P}$ y K $(70 \mathrm{~kg}$ de $\mathrm{P}_{2} \mathrm{O}_{5}$ y $375 \mathrm{~kg}$ de $\mathrm{K}_{2} \mathrm{O} / \mathrm{ha}$ ), estos valores superaron a los encontrados por Rojas (1993), quien obtuvo 0,64 y $0,20 \mathrm{~m}$ de altura y circunferencia respectivamente, a los cuatro meses de edad en el mismo cultivar. A los siete meses obtuvo valores de 1,88 y $0,46 \mathrm{~m}$, también más bajos que los de este estudio ( 2,68 y $0,65 \mathrm{~m})$. Por el contrario, los valores de altura a los siete meses de edad fueron menores que los medidos $(3,49 \mathrm{~m})$ por González (1999), en el mismo cultivar Curraré. 


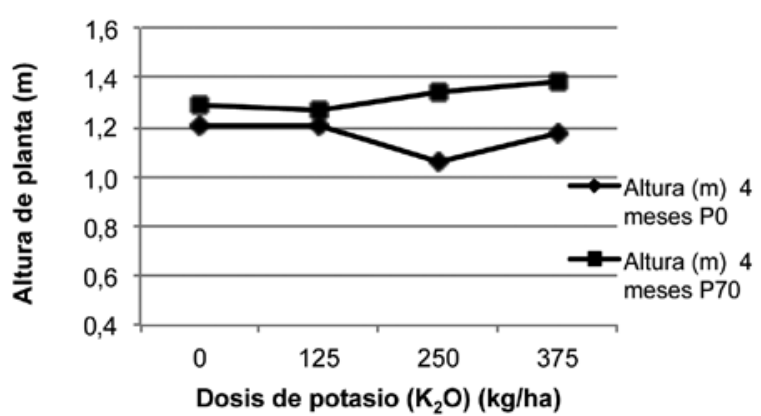

Figura 1 . Representación de la interacción de fósforo-potasio en altura del pseudotallo de plátano a los cuatro meses de edad. Cosecha 2009. La Fortuna, San Carlos, Costa Rica. Octubre del 2010.

Respecto a la cosecha de 2010, hubo diferencia estadística al $\mathrm{K}$ en las variables de crecimiento AP y GP a los cuatro y siete meses de edad, en el experimento $\mathrm{K}^{*} \mathrm{~S}$, con p-valor entre 0,033 y 0,0004 ; en las Figuras 3 y 4 se observa el comportamiento de los valores, donde tanto la altura y la circunferencia fueron más altas en la cosecha de 2009 respecto a la de 2010; sin embargo, el rendimiento fue más alto en el 2010, quizás por el ataque de bacteriosis en el cultivo cosechado en el 2009. En el año 2010 hubo respuesta estadística significativa ( $p$-valor $\leq 0,05$ ) en los dos ensayos en las variables de rendimiento $\mathrm{PR}, \mathrm{LD}$ y $\mathrm{CD}$. En las Figuras 5,6 y 7 , se aprecia la tendencia de los valores; en las dos primeras variables resulta una tendencia lineal con p-valor $\leq 0,01$, mientras que para $\mathrm{CD}$ la tendencia es

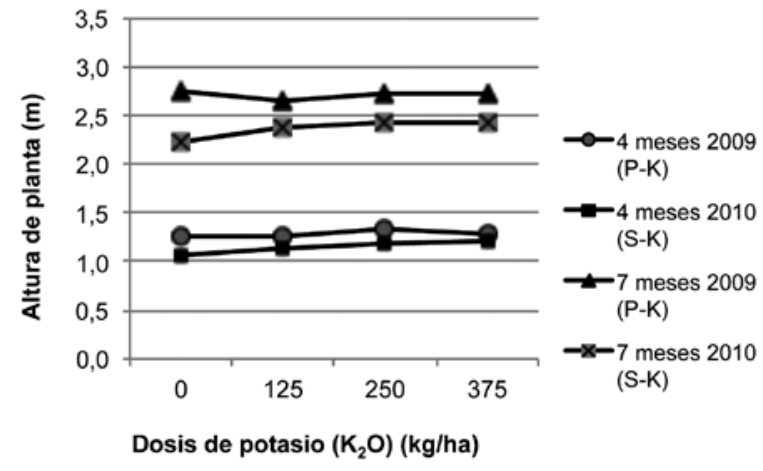

Figura 3. Comportamiento de la altura del pseudotallo de la planta de plátano a los cuatro y siete meses de edad. Ensayos azufre-potasio y fósforo-potasio. La Fortuna, San Carlos, Costa Rica. Octubre del 2010.

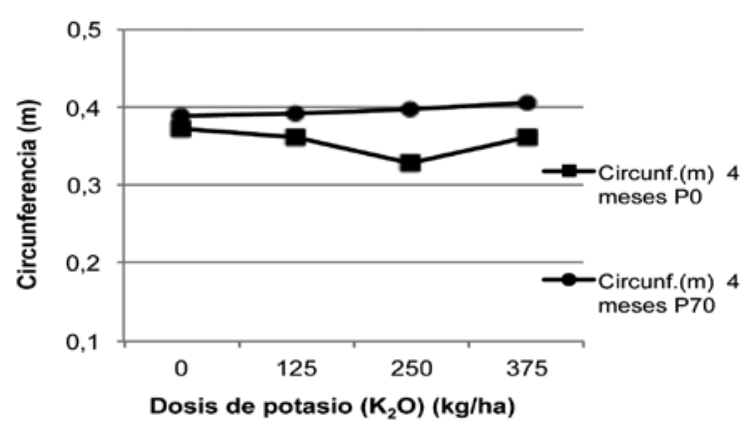

Figura 2. Representación de la interacción de fósforo-potasio en la circunferencia de la base del pseudotallo de planta de plátano a los cuatro meses de edad. Cosecha 2009, La Fortuna, San Carlos, Costa Rica. Octubre del 2010.

cúbica en el experimento $\mathrm{K} * \mathrm{P}$ con $\mathrm{p}$-valor $=0,0448$; por el contrario, en el 2009 en el ensayo $\mathrm{K} * \mathrm{~S}$ la tendencia de CD es lineal y en el 2010 cuadrática (Figura 7).

Los calibres de dedos aunque no siguen una tendencia conforme a la dosis del nutrimento aplicado, en sentido general son normales para la variedad de plátano estudiada; los mismos son mayores que los encontrados por González (1999) con promedio de 56,2 en la misma variedad pero con 3200 plantas por hectárea y que los obtenidos por Lardizabal (2007) en el material "Curraré enano" con un promedio de 52 treinta y dozavos de pulgada, este autor indica que valores menores a estos caracterizan a frutos de bajo peso y calidad.

Aunque la variable $\mathrm{N}^{\circ} \mathrm{DR}$ no presentó diferencia estadística a la aplicación de K, es importante resaltar

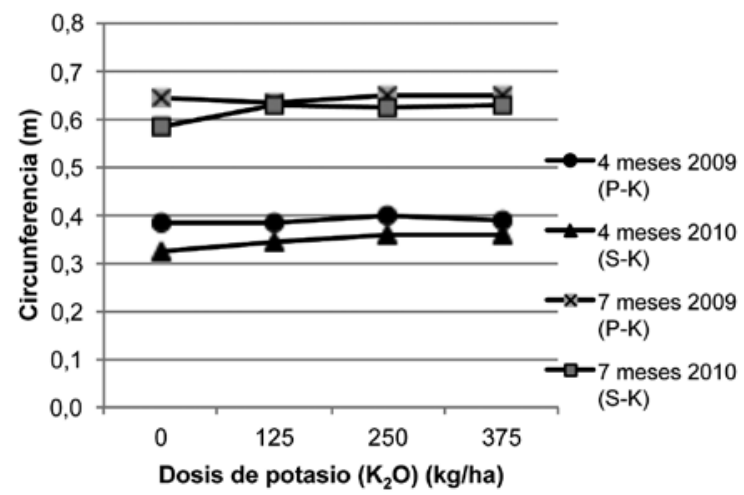

Figura 4. Comportamiento de la circunferencia del pseudotallo de la planta de plátano a los cuatro meses y siete meses de edad. Ensayos azufre-potasio y fósforo-potasio. La Fortuna, San Carlos, Costa Rica. Octubre del 2010. 


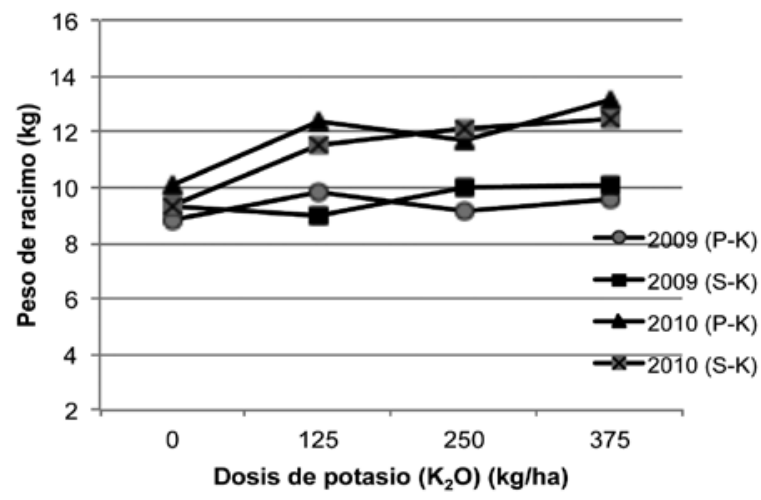

Figura 5. Comportamiento del peso de racimo de plátano. Ensayos azufre-potasio y fósforo-potasio. La Fortuna, San Carlos, Costa Rica. Octubre del 2010.

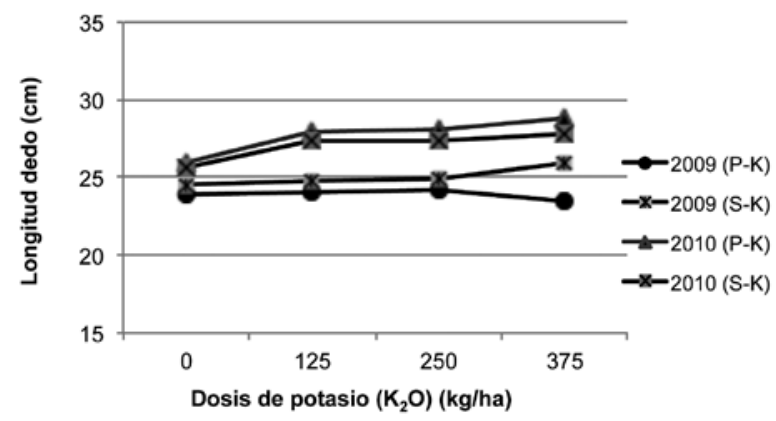

Figura 6. Comportamiento de la longitud del dedo central de la segunda mano del racimo de plátano. Ensayos azufre-potasio y fósforo-potasio. La Fortuna, San Carlos, Costa Rica. Octubre del 2010.

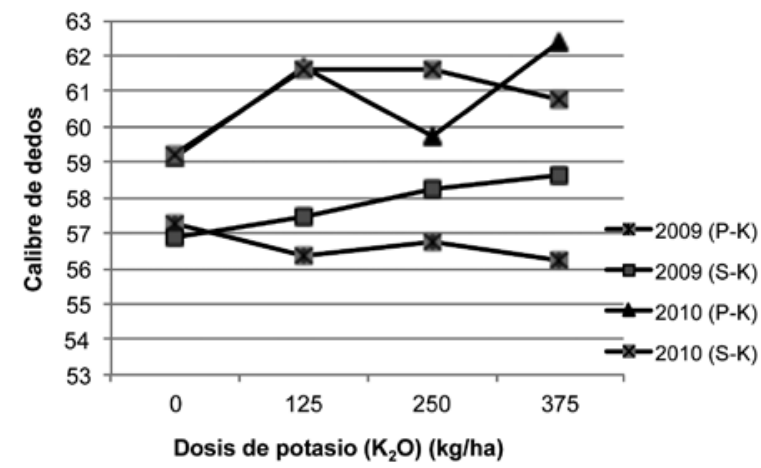

Figura 7. Comportamiento de calibre del dedo central de la segunda mano del racimo de plátano. Ensayos azufre-potasio y fósforo-potasio. La Fortuna, San Carlos, Costa Rica. Octubre del 2010. que en la cosecha de 2010 del ensayo $\mathrm{K} * \mathrm{~S}$ hubo una tendencia lineal positiva, según la dosis de $\mathrm{K}$ aplicada. A pesar que en esta cosecha el peso del racimo fue mayor que en la de 2009 (Figura 5), el $N^{\circ} \mathrm{DR}$ fue menor (Figura 8); esto indica que el rendimiento del racimo no solo está definido por esta variable, sino también por otras como PR, LD y CD (González 1999, Hernández et al. 2007), lo que confirma el hecho que las variables $\mathrm{PR}, \mathrm{LD}$ y $\mathrm{CD}$ son mayores en la cosecha de 2010, donde el rendimiento es mayor, que en la cosecha de 2009 (Figuras 5, 6, 7 y 8). La interacción entre el $\mathrm{K}$ y el $\mathrm{P}$ fue estadísticamente significativa $(\mathrm{p}=0,0008)$ para $\mathrm{N}^{\circ} \mathrm{DR}$ (Figura 9); el PR además de presentar diferencia estadística al uso de K en el 2010, también lo fue la interacción K-P (Figura 10).

La deficiencia de $\mathrm{K}$ afecta el peso total del racimo y el número de manos por racimo ( ${ }^{\circ} \mathrm{MR}$ ) (Mena 1997). En la discusión anterior se comenta la importancia en el incremento del PR cuando hay suficiente $\mathrm{K}$, no obstante respecto a la variable $\mathrm{N}^{\circ} \mathrm{MR}$ no se encontró respuesta a ninguno de los tres elementos nutricionales utilizados, es posible que esta variable, esté controlada por el material genético, como lo indican Orozco y Chaverra (1999), con posibilidad de variación únicamente por efecto hídrico y nutricional principalmente durante el inicio de crecimiento del cultivo. No obstante, González (1999) encontró entre 6,36 y 6,44 manos por racimo con 3200 plantas por hectárea, pero al reducir la densidad de plantas a 1800 , el $\mathrm{N}^{\circ} \mathrm{MR}$ subió a 7,41 en el cultivar Curraré. Este autor atribuye el aumento en manos al vigor de las plantas por menor crecimiento del follaje por planta. En el

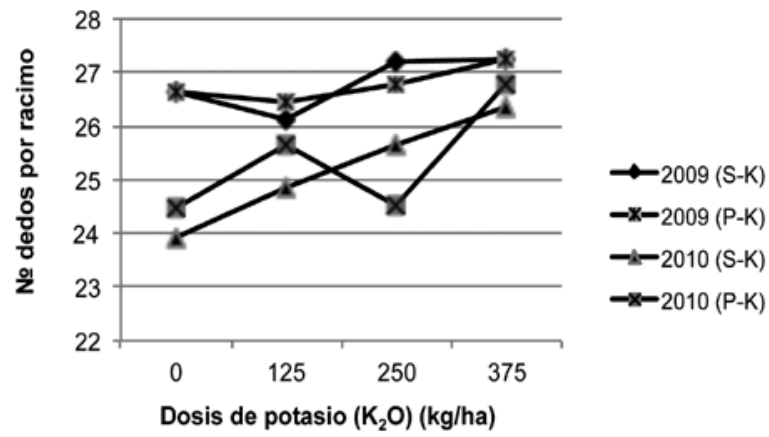

Figura 8. Comportamiento de número de dedos por racimo de plátano. Ensayos azufre-potasio y fósforo-potasio. La Fortuna, San Carlos, Costa Rica. Octubre del 2010. 


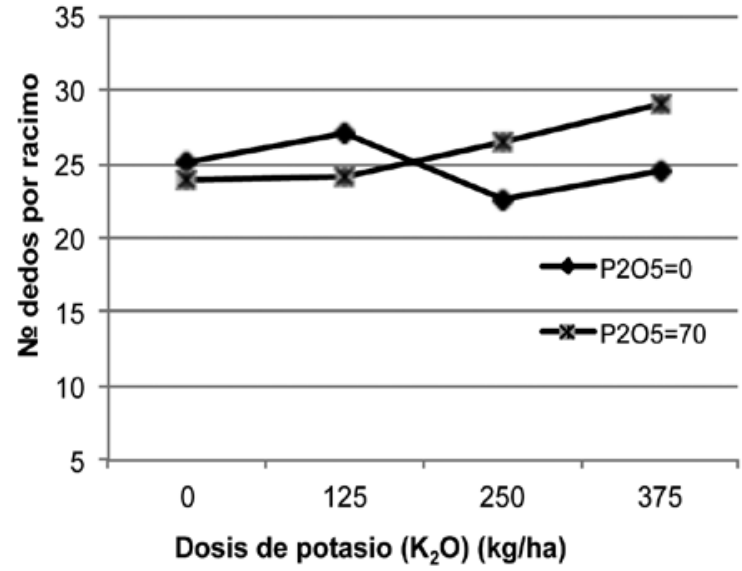

Figura 9. Representación de la interacción de fósforo-potasio en $\mathrm{N}^{\circ}$ de dedos por racimo de plátano. Cosecha 2010. La Fortuna, San Carlos, Costa Rica. Octubre del 2010.

estudio que nos compete hubo un valor frecuente de seis manos en la mayoría de los racimos.

En suelos con materiales de origen volcánicos, con alto contenido de bases y valores altos de $\mathrm{K}$ respecto al magnesio, Bolaños et al. (2002) indican que no hubo respuesta al N-P-K; los resultados de P y K en la cosecha 2009 tienen similitud con los indicados por estos autores. En cambio, en la cosecha 2010 los resultados positivos obtenidos con la aplicación de $\mathrm{K}$ difieren de los anteriores; caso contrario, con el $\mathrm{P}$ cuyos resultados positivos fueron pocos en las dos cosechas. En la cosecha de 2010, no hubo respuesta a la aplicación de $\mathrm{P}$ en ninguna de las variables, excepto algunas interacciones con el $\mathrm{K}$ para $\mathrm{PR}$ y $\mathrm{N}^{\circ} \mathrm{MR}$, mientras que en el 2009 hubo respuesta únicamente en altura y circunferencia de pseudotallo a los cuatro meses de edad.

Información de Vázquez et al. (2005), muestra que el fósforo es importante en la formación de raíces y del racimo, y que su deficiencia disminuye el ritmo de producción de hojas; opinión similar tiene Mena (1997) respecto al crecimiento de raíces; a pesar de lo anterior, este último menciona que la respuesta de las musáceas a la aplicación de $\mathrm{P}$ es cuestionada. Del mismo modo, López (2002) informa que diversos estudios no han encontrado respuesta del plátano a la fertilización con P, resultados similares obtuvieron Espinosa y Belalcázar (1998). Por otro lado, Marín

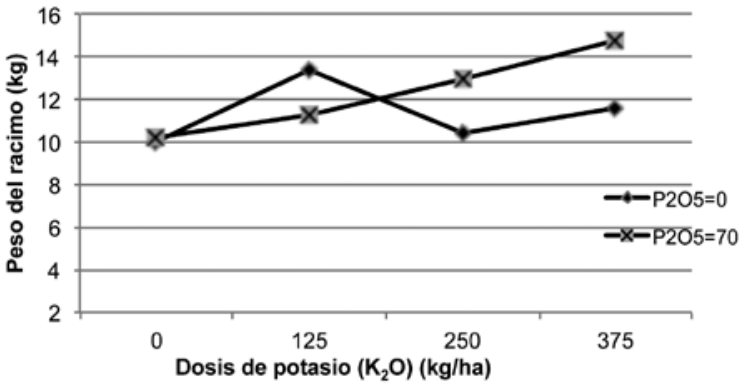

Figura 10. Representación de la interacción de fósforo-potasio en peso del racimo de plátano. Cosecha 2010. La Fortuna, San Carlos, Costa Rica. Octubre del 2010.

y De Roberti (1992) menciona que la aplicación de $\mathrm{P}$ al suelo no altera los contenidos de N-P-K en las hojas en ninguna etapa, ni eleva el rendimiento. La absorción total de $\mathrm{P}$ al momento de la cosecha fue baja (Cuadro 1); según los análisis de suelos al inicio de la siembra la cantidad de P fue estimada entre 9 y $10 \mathrm{~kg} /$ ha. Por consiguiente, de acuerdo con los resultados de absorción de $\mathrm{P}$, de los análisis de suelos y la respuesta encontrada a su aplicación, se concluye que para que el cultivo de plátano manifieste respuesta a la aplicación de $\mathrm{P}$, el suelo debería ser muy pobre en este elemento.

En este estudio, tampoco se encontraron cambios por la aplicación de $\mathrm{P}$ al suelo en el contenido de ese elemento en las hojas muestreadas al inicio de la floración del cultivo (Cuadro 2). Es evidente que la concentración de varios elementos, fue mayor o igual en la cosecha 2010, que en la de 2009, excepto el K que fue más bajo (Cuadro 2), lo que coincide con lo indicado por Mena (1997), que el potasio disminuye en las hojas a partir de la floración debido a que es translocado al racimo, lo que le confiere mayor peso.

La concentración de nutrimentos en los diferentes órganos o estructuras se presentan en el Cuadro 3. El pinzote fue la estructura con mayor concentración de $\mathrm{K}, \mathrm{P}$ y S, seguido de las hojas con alta concentración de nutrimentos mayormente de N, P y S. El K fue más bajo en las hojas al momento de la cosecha que durante la floración (Cuadros 2 y 3). Estos resultados son confirmados por Mena (1997) en el sentido que este autor indica que el $\mathrm{K}$ es translocado desde las hojas al racimo para el llenado y aumento de peso del mismo. Otros elementos que resultaron con comportamiento similar al $\mathrm{K}$ son el $\mathrm{N}$, P y S, resultado que es más 
Cuadro 1. Absorción de nutrimentos al momento de la cosecha y porcentaje de absorción de la parte comercial y no comercial del cultivo de plátano en dos cosechas 2009 y 2010. La Fortuna, San Carlos, Costa Rica. Octubre del 2010.

\begin{tabular}{|c|c|c|c|c|c|c|c|c|}
\hline \multirow{3}{*}{ Elemento } & \multicolumn{4}{|c|}{ Absorción } & \multicolumn{4}{|c|}{ Porcentaje de extracción } \\
\hline & \multicolumn{2}{|c|}{ Total (kg/ha) } & \multicolumn{2}{|c|}{$\%$ por elemento } & \multicolumn{2}{|c|}{ Comercial (dedos) } & \multicolumn{2}{|c|}{ No comercial* } \\
\hline & 2009 & 2010 & 2009 & 2010 & 2009 & 2010 & 2009 & 2010 \\
\hline $\mathrm{P}$ & 10,21 & 10,83 & 1,60 & 2,44 & 41,79 & 67,55 & 58,21 & 32,45 \\
\hline $\mathrm{K}$ & 452,39 & 237,73 & 70,78 & 53,56 & 15,30 & 57,24 & 84,70 & 42,76 \\
\hline $\mathrm{S}$ & 6,51 & 7,44 & 1,02 & 1,68 & 24,86 & 46,69 & 75,14 & 53,31 \\
\hline $\mathrm{Fe}$ & 4,89 & 5,64 & 0,76 & 1,27 & 1,94 & 1,94 & 98,06 & 98,06 \\
\hline $\mathrm{Cu}$ & 0,03 & 0,07 & 0,005 & 0,17 & 19,81 & 42,44 & 80,19 & 57,56 \\
\hline $\mathrm{Zn}$ & 0,20 & 0,17 & 0,03 & 0,04 & 14,06 & 47,75 & 85,94 & 52,25 \\
\hline Mn & 1,94 & 1,53 & 0,30 & 0,34 & 0,61 & 0,01 & 99,39 & 99,99 \\
\hline B & 0,08 & 0,11 & 0,01 & 0,02 & 15,62 & 52,55 & 84,38 & 47,45 \\
\hline
\end{tabular}

* raíz, cormo, seudotallo, hojas, raquis y pinzote.

evidente en la cosecha de 2010 que en la de 2009; quizás esta es una razón por la cual el peso del racimo (PR) fue mayor en la cosecha 2010 (pesos promedio entre 9,35 y $13,17 \mathrm{~kg}$ ) que en la cosecha de 2009. Los pesos de racimos encontrados en este estudio, son similares a los obtenidos por González (1999) quien reporta peso por racimo de $10,98 \pm 2,18 \mathrm{~kg}$ y a los alcanzados por Rojas (1993) con peso de $11,37 \mathrm{~kg}$, ambos autores trabajaron con el cultivar Curraré.

La concentración de nutrimentos en las hojas y el rendimiento del cultivo, se relacionan; según Marín y De Roberti (1992), la concentración foliar de P debe encontrarse entre 0,20 a $0,27 \%$, para obtener buenos rendimientos en los suelos de la serie Chama en Venezuela. En nuestro medio el P presentó valores de 0,215 y $0,223 \%$ en la cosecha de 2009 y en la 2010 valores de 0,228 y $0,232 \%$ para los tratamientos P0 y P70, respectivamente (Cuadro 2); lo que indica que bajo los criterios de Marín y De Roberti (1992) la hoja tiene suficiente concentración foliar incluyendo el tratamiento sin P; posiblemente por la concentración normal en las hojas cuando no se aplicó fósforo ni azufre (P0 y S0) fue que no hubo respuesta a la aplicación de esos dos elementos.

Igual que la concentración de $\mathrm{P}$ en las hojas, la de azufre (S) no aumentó significativamente con el aporte de ese elemento al suelo (Cuadro 2); asimismo, no hubo respuesta en las variables de crecimiento y rendimiento con la dosis aplicada, excepto para la longitud del dedo central de la segunda mano cuando se aplicaron $30 \mathrm{~kg}$ de S/ha en la cosecha de 2010, sin afectar el peso del racimo. Espinosa y Belalcázar (1998) encontraron mejor respuesta al potasio cuando se agregó $\mathrm{S}$, en un suelo pobre en este elemento. Según Palencia et al. (2006) el plátano absorbe $30 \mathrm{~kg} / \mathrm{ha}$ de $\mathrm{S}$ y que la respuesta a la fertilización de cualquier nutrimento dependerá de las condiciones del suelo. En este estudio, la absorción total de S fue baja (Cuadro 1) respecto a lo reportado por Palencia et al. (2006). Asimismo el suelo donde se hizo la primera siembra, período 2008-2009, tenía bajo contenido, con un valor de $4 \mathrm{mg} / \mathrm{l}$, lo que representó alrededor de $9 \mathrm{~kg} / \mathrm{ha}$. Sin embargo, esta cantidad parece suficiente para el cultivo que en ese período absorbió $6,51 \mathrm{~kg}$ de $\mathrm{S} / \mathrm{ha}$, puesto que la concentración en lámina de las hojas fue similar con y sin aplicación de S (Cuadro 2). Por el contrario, en el año 2010, el análisis de suelo dio como resultado $14 \mathrm{mg} / \mathrm{l}$, valor que puede ser más que suficiente para satisfacer las necesidades de absorción de las plantas de plátano que en ese año fue de 7,44 $\mathrm{kg}$ de $\mathrm{S} / \mathrm{ha}$. Aunque según Bornemisza (1990), el S puede estar deficiente en suelos volcánicos cuando son abonados 
Cuadro 2. Contenido de elementos en la lámina de la hoja de plátano muestreada al inicio de la floración (siete meses), en dos cosechas de plátano bajo fertilización P, K y S. La Fortuna, San Carlos, Costa Rica. Octubre del 2010.

\begin{tabular}{|c|c|c|c|c|c|c|c|c|c|c|c|}
\hline \multicolumn{12}{|c|}{ Resultados de análisis foliar. Cosecha 2009} \\
\hline \multirow[t]{2}{*}{ Niveles de $\mathrm{P}_{2} \mathrm{O}_{5}$} & \multicolumn{6}{|c|}{$\%$} & \multicolumn{5}{|c|}{$\mathrm{mg} / \mathrm{kg}$} \\
\hline & $\mathbf{N}$ & $\mathbf{P}$ & $\mathbf{C a}$ & Mg & $\mathbf{K}$ & $\mathbf{S}$ & $\mathbf{F e}$ & $\mathbf{C u}$ & Zn & Mn & B \\
\hline 0 & 3,81 & 0,215 & 0,84 & 0,39 & 3,17 & 0,23 & 96,75 & 8,25 & 15,75 & 385,3 & 7,75 \\
\hline 70 & 3,84 & 0,223 & 0,85 & 0,41 & 3,21 & 0,24 & 105,25 & 8,00 & 16,75 & 449,5 & 7,50 \\
\hline \multicolumn{12}{|l|}{ Niveles de $\mathrm{K}_{2} \mathrm{O}$} \\
\hline 0 & 3,81 & 0,21 & 0,75 & 0,37 & $\mathbf{3 , 0 3}$ & 0,22 & 87,50 & 8,00 & 14,50 & 305,0 & 7,00 \\
\hline 125 & 3,81 & 0,22 & 0,85 & 0,42 & 3,24 & 0,24 & 105,50 & 8,00 & 16,50 & 457,5 & 7,50 \\
\hline 250 & 3,78 & 0,23 & 0,92 & 0,42 & 3,34 & 0,24 & 100,00 & 8,50 & 17,00 & 422,0 & 8,00 \\
\hline 325 & 3,92 & 0,23 & 0,87 & 0,41 & 3,16 & 0,24 & 111,00 & 8,00 & 17,00 & 485,0 & 8,00 \\
\hline \multicolumn{12}{|l|}{ Niveles de $S$} \\
\hline 0 & 3,88 & 0,22 & 0,81 & 0,42 & 3,10 & $\mathbf{0 , 2 3 5}$ & 95,0 & 8,25 & 16,3 & 459 & 7,5 \\
\hline 30 & 3,90 & 0,21 & 0,85 & 0,42 & 3,04 & 0,24 & 88,5 & 7,75 & 16,3 & 462 & 7,0 \\
\hline \multicolumn{12}{|c|}{ Resultados de análisis foliar. Cosecha 2010} \\
\hline Niveles de $\mathrm{P}_{2} \mathrm{O}_{5}$ & \multicolumn{6}{|c|}{$\%$} & \multicolumn{5}{|c|}{$\mathrm{mg} / \mathrm{kg}$} \\
\hline & $\mathbf{N}$ & $\mathbf{P}$ & $\mathbf{C a}$ & Mg & $\mathbf{K}$ & $\mathbf{S}$ & $\mathrm{Fe}$ & $\mathrm{Cu}$ & $\mathrm{Zn}$ & Mn & B \\
\hline 0 & 4,32 & 0,228 & 0,85 & 0,58 & 2,98 & 0,27 & 75,50 & 9,75 & 18,75 & 590 & 9,50 \\
\hline 70 & 4,36 & 0,233 & 0,88 & 0,62 & 2,84 & 0,26 & 71,75 & 9,25 & 18,25 & 633 & 9,25 \\
\hline \multicolumn{12}{|l|}{ Niveles de $\mathrm{K}_{2} \mathrm{O}$} \\
\hline 0 & 4,39 & 0,23 & 0,90 & 0,65 & 2,77 & 0,27 & 70,00 & 10,00 & 18,50 & 447 & 9,50 \\
\hline 125 & 4,40 & 0,23 & 0,84 & 0,61 & 2,90 & 0,27 & 72,50 & 9,00 & 18,50 & 611 & 9,50 \\
\hline 250 & 4,30 & 0,23 & 0,85 & 0,58 & 2,95 & 0,27 & 73,00 & 9,00 & 17,50 & 638 & 9,50 \\
\hline 325 & 4,28 & 0,24 & 0,86 & 0,58 & 3,02 & 0,26 & 79,00 & 10,00 & 19,50 & 750 & 9,00 \\
\hline \multicolumn{12}{|l|}{ Niveles de $S$} \\
\hline 0 & 4,43 & 0,23 & 0,88 & 0,62 & 2,76 & 0,263 & 71,0 & 9,25 & 17,5 & 688 & 9,50 \\
\hline 30 & 4,41 & 0,23 & 0,87 & 0,62 & 2,89 & 0,26 & 70,5 & 9,25 & 19,0 & 739 & 9,75 \\
\hline
\end{tabular}

con suficientes cantidades de $\mathrm{P}$ y $\mathrm{B}$, en el caso que nos compete se aplicaron $70 \mathrm{~kg}$ de $\mathrm{P}_{2} \mathrm{O}_{5} /$ ha y los productores normalmente fertilizan con alto contenido de P. A pesar de esta posible afectación de disponibilidad de $\mathrm{S}$, no hubo respuesta en el rendimiento y calidad del plátano a la aplicación de $30 \mathrm{~kg}$ de $\mathrm{S} / \mathrm{ha}$.

$\mathrm{La}$ alta absorción de $\mathrm{K}$ en la primera cosecha $(452,4 \mathrm{~kg} / \mathrm{ha})$, no es la esperada, a pesar de que concuerda con lo expuesto por Palencia et al. (2006), al expresar que el plátano puede extraer 440 kilogramos.
La absorción de la segunda cosecha fue de 237,73 $\mathrm{kg}$ de $\mathrm{K} / \mathrm{ha}$ (alrededor de $285 \mathrm{~kg}$ de $\mathrm{K}_{2} \mathrm{O}$ ), cuando se considera la eficiencia del elemento aplicado en el suelo, concuerda con la dosis alta usada en este experimento. De igual modo, tiene cierta similitud con las dosis indicadas por autores como López (2002) y Combatt et al. (2004) quienes proponen dosis entre 250 y $300 \mathrm{~kg}$ de $\mathrm{K}_{2} \mathrm{O} / \mathrm{ha}$; Espinosa y Belalcázar (1998), indican que el plátano requiere de 210 a $280 \mathrm{~kg}$ de $\mathrm{K}_{2} \mathrm{O} / \mathrm{ha}$. 
Cuadro 3. Contenido promedio de los elementos analizados en las estructuras de la planta plátano al momento de la cosecha bajo fertilización P, K y S. La Fortuna, San Carlos, Costa Rica. Octubre del 2010.

\begin{tabular}{lccccccccc}
\hline \multirow{2}{*}{ Estructura } & \multicolumn{10}{c}{ Cosecha 2009 } \\
\cline { 2 - 11 } & $\mathbf{9}$ & $\mathbf{P}$ & $\mathbf{K}$ & $\mathbf{S}$ & $\mathbf{F e}$ & $\mathbf{C u}$ & $\mathbf{Z n}$ & $\mathbf{M n}$ & $\mathbf{B}$ \\
\cline { 2 - 11 } Lámina de hoja & 3,04 & 0,22 & $\mathbf{2 , 6 1}$ & 0,21 & 108 & 3,75 & 16,25 & 818 & 10,75 \\
Raquis & 1,03 & 0,08 & 5,20 & 0,07 & 289 & 2,25 & 14,50 & 241 & 7,00 \\
Pinzote & 1,80 & 0,28 & 10,69 & 0,19 & 300 & 1,50 & 17,50 & 66 & 19,50 \\
Dedos & 0,40 & 0,07 & 1,14 & 0,03 & 13 & 1,00 & 4,50 & 1,51 & 2,00 \\
Tallo & 0,78 & 0,07 & 6,81 & 0,06 & 869 & 3,50 & 35,00 & 246 & 12,00 \\
Cormo & 0,64 & 0,05 & 4,65 & 0,03 & 293 & 3,00 & 20,50 & 88 & 5,00 \\
Raíz & 1,13 & 0,07 & 3,60 & 0.08 & 5.34 & 15,00 & 23,25 & 230 & 15,00 \\
\hline & & & & & Cosecha 2010 & & & \\
\hline Lámina de hoja & 2,69 & 0,18 & $\mathbf{1 , 7 7}$ & 0,21 & 298 & 8,00 & 23,67 & 1.243 & 12,67 \\
Raquis & 0,83 & 0,06 & 1,33 & 0,09 & 88 & 6,33 & 18,00 & 273 & 10,33 \\
Pinzote & 1,82 & 0,23 & 10,2 & 0,36 & 129 & 2,67 & 16,00 & 86 & 29,67 \\
Dedos & 0,52 & 0,07 & 1,30 & 0,03 & 8 & 3,00 & 7,67 & 0,01 & 5,33 \\
Tallo & 0,70 & 0,06 & 2,67 & 0,07 & 188 & 11,67 & 41,00 & 221 & 12,33 \\
Cormo & 0,75 & 0,05 & 1,53 & 0,04 & 506 & 9,00 & 13,33 & 154 & 6,00 \\
Raíz & 1,14 & 0,08 & 4,02 & 0,08 & 8.22 & 21,33 & 31,67 & 371 & 26,67 \\
\hline
\end{tabular}

\section{LITERATURA CITADA}

Barrientos, O; Chaves, G. 2008. Región Huetar Norte. Oferta exportada actual y oferta potencial de productos agropecuarios alternativos. SEPSA y PROCOMER. San José, C.R. p. 83-87 (en línea). Consultado 30 jun. 2009. Disponible en www.infoagro.go.cr/ documentospdf/Huetar_NorteLibro-final.pdf

Belalcázar, S. 1991. El cultivo del plátano (Musa AAB) en el trópico. Colombia, IICA. 376 p.

Bolaños, MM; Celis, LD; Morales, H. 2002. Fertilización y residualidad de nutrimentos, en el cultivo de plátano (Musa $A A B$ ) en un andisol de Quindío, Colombia. In Memorias XV Reunión Acorbat. Cartagena de Indias, Colombia: Asociación de Bananeros de Colombia AUGURA. Medellín, Colombia. p. 436-440.

Bornemisza, E. 1990. Problemas del azufre en suelos y cultivos de Mesoamérica. San José, Costa Rica, Editorial de la Universidad de Costa Rica. p. 24-25.

Combatt, EM; Martínez, G; Barrera, JL. 2004. Efecto de la interacción de $\mathrm{N}$ y $\mathrm{K}$ sobre las variables de rendimiento del cultivo de plátano (Musa AAB cv
Simmonds) en San Juan de Uraba-Antioquia. Rev. Temas Agrarios 9(1):5-12.

Espinosa, J; Belalcázar, S. 1998. Fertilization of plantain in high densities. Photash and Phosphate Institute, Latin America Office and Colombian Institute of Agronomic Research, Armenia - Colombia (en línea). Consultado 20 dic. 2006. Disponible en http://www. ipni.net/publication/bci.nsf/0/5CA8DBC8F51 A6A4 385257BBA006D6110/\$FILE/Better\%20Crops\%20 International\%202000-1\%20p16.pdf

González, H. 1999. Evaluación de tres métodos y cuatro densidades de siembra en la producción de la segunda generación (hijos) del plátano (Musa AAB) cv. "Curraré" en la zona de San Carlos, Costa Rica. Tesis de Lic. Costa Rica, Instituto Tecnológico de Costa Rica. 74 p.

Guerrero, M. 2010. Guía técnica del cultivo del plátano. Programa MAG-CENTA-Frutales. El Salvador, CENTA. 24 p.

Hernández, Y; Marín, M; García, J. 2007. Respuesta en el rendimiento del plátano (Musa AAB cv. Hartón) en función de la nutrición mineral y su ciclo fenológico. Parte I. Crecimiento y producción. Rev. Fac. Agronomía (LUZ) 24:607-626. 
InfoStat. 2008. InfoStat versión 2008. Manual del usuario. Grupo InfoStat, FCA, Universidad Nacional de Córdoba, Argentina, Editorial Brujas. 336 p.

Lardizabal, R. 2007. Producción de plátano en alta densidad. EDA (Entrenamiento y Desarrollo de Agricultores) (en línea). Consultado 3 set. 2009. Disponible en http://www.mcahonduras.hn/documentos/ PublicacionesEDA/Manuales\%20de\%20produccion/ EDA_Manual_Produccion_Platano_05_07.pdf

López, OR. 2002. Manual de producción de plátano basado en la experiencia de Zamorano. Tesis Lic. Honduras, El Zamorano. p. 35.

Marín, M; De Roberti, R. 1992. Importancia del análisis foliar en la evaluación de la fertilidad de suelos en Venezuela. Universidad del Zulia. Maracaibo. Rev. Fac. Agronomía (LUZ) 9:1-15.

Mena, VJ. 1997. Manejo agronómico y levantamiento de malezas prevalecientes en una plantación de plátano "Curraré" en la finca La Vega, Región Huetar Norte. Tesis Lic. San Carlos, Costa Rica, Instituto Tecnológico de Costa Rica. 54 p.

Navarro, JM; Arias, HV; Caravaca, VP; Solano, V. 2009. Situación actual del mercado nacional e internacional. Boletín informativo del plátano. CNP (Consejo Nacional de Producción). SIIM (Servicio de Información e Inteligencia de Mercados). San José, C.R. Boletín $\mathrm{N}^{\circ}$ 1, 2009 (en línea). Consultado 20 nov. 2009. Disponible en http://web.cnp.go.cr/images/ SIIM/analisis/frutas/platano/2009/Platano_01-09.pdf

Orozco, R; Chaverra, C. 1999. Curso de actualización tecnológica en el cultivo del plátano con énfasis en postcosecha. CORPOICA (Corporación Colombiana de Investigación Agropecuaria) y PRONATTA (Programa Nacional de Transferencia de Tecnología) (en línea). Consultado 6 ago. 2008. Disponible en http://www.docstoc.com/docs/3176765/CURSO-

DE-ACTUALIZACION-TECNOLOGICA-EN-ELCULTIVO-DEL-PLATANO-CON

Palencia, GE; Gómez, R; Martín, JE. 2006. Manejo sostenible del cultivo de plátano. Colombia, CORPOICA (Corporación Colombiana de Investigación Agropecuaria). p. 16-18.

Rojas, E. 1993. Influencia del material de siembra sobre la producción de plátano (Musa AAB) en la zona de San Carlos, Costa Rica. Tesis Lic. Costa Rica, Instituto Tecnológico de Costa Rica. 75 p.

Rodríguez, M; Guerrero, M. 2002. Guía técnica del cultivo del plátano. No. 4. CENTA (Centro Nacional de Tecnología Agropecuaria y Forestal Enrique Álvarez Córdoba). El Salvador (en línea). Consultado 22 ago. 2009. Disponible en http://www.centa.gob.sv/docs/ guias/frutales/Platano.pdf

Sancho, H. 1999. Curvas de absorción de nutrientes: importancia y uso en los programas de fertilización. Informaciones agronómicas No. 36:11-13 (en línea). Consultado 1 dic. 2006. Disponible en http://www. ipni.net/publication/ia-lahp.nsf/0/765C8D66598A4 91B852579A3007A3289/\$FILE/Curvas\%20de\%20 Absorci\%C3\%B3n.pdf

Vázquez, R; Romero, A; Figueroa, J; Munro, D. 2005. Paquete tecnológico para el cultivo de plátano. Gobierno del Estado de Colima, México. p. 23. 
